\title{
At the Junction of Immunity and Barrier Function: The Immunomodulatory Protein TL1A May Also Regulate Intestinal Permeability
}

\author{
Giorgos Bamias ${ }^{1}$
}

Published online: 16 May 2019

(c) Springer Science+Business Media, LLC, part of Springer Nature 2019

The current concept of inflammatory bowel disease (IBD) pathogenesis states that one of the earlier events in disease development is the disruption of the barrier function of the intestinal epithelial layer. This most probably is the result of harmful environmental pressure and, in turn, facilitates the increased influx of bacterial components such as endotoxin into the portal vein, thus triggering the persistent stimulation of innate and adaptive immune mechanisms through activation of immune receptors expressed on reticuloendothelial cells in the liver and elsewhere. The importance of paracellular permeability as a biomarker is supported by its increase that is observed in first-degree relatives of patients with IBD and in patients with quiescent IBD, with its association with ongoing symptomatology despite mucosal healing, and its predictive value for subsequent disease flares [1]. That being said, the relation between paracellular permeability and the intestinal transport of bacterial toxins has not been confirmed in intact systems, although paracellular permeability and endotoxin transport are strongly associated. Thus, understanding the mechanisms that underlie loss of barrier function would be of great importance, as it may enable the early therapeutic intervention in patients with IBD.

Following the successful application of anti-tumor necrosis factor (TNF)- $\alpha$ targeted therapies for the treatment of IBD, the contribution of members of the TNF and TNFR superfamilies (TNFSF and TNFRSF, respectively) toward intestinal inflammation has been the focus of intensive research. Among those proteins, TNF-like cytokine 1A (TL1A, TNFSF15) and its functional receptor deathdomain receptor 3 (DR3, TNFRSF25) have emerged as important molecular mediators of mucosal homeostasis

Giorgos Bamias

gbamias@gmail.com; gbamias@med.uoa.gr

1 GI-Unit, 3rd Academic Department of Internal Medicine, Sotiria Hospital, National and Kapodistrian University of Athens, 152 Mesogeion Avenue, 11527 Athens, Greece and inflammation [2]. Converging lines of evidence support the function of TL1A/DR3 as a co-stimulatory system that participates in the shaping and execution of immunological responses, affecting both effector and regulatory $\mathrm{T}$ cell function and also interfering with innate immunity. Nevertheless, due to these broad and multifaceted functions, the exact participation of TL1A and DR3 in the pathogenesis of Crohn's disease (CD) and ulcerative colitis (UC) still remains largely undefined.

In this issue of Digestive Diseases and Sciences, Yang et al. [3] propose a novel function for TL1A as a regulator of intestinal epithelial barrier integrity. The authors tested the effect of constitutive myeloid-specific overexpression of TL1A and found that, in response to dextran sodium sulfate (DSS) administration, TL1A-Tg mice developed significantly severer colitis and more marked impairment of epithelial barrier function. The latter was signified by increased bacteria present in mesenteric lymph nodes, paracellular permeability to a small solute, and circulating concentrations of lipopolysaccharide (LPS). Those effects were linked to a rearrangement of the expression of proteins associated with tight junction formation, including claudins $1-3$, zona occludens (ZO)-1, occludin, and junction adhesion molecules (JAM)s. The findings were recapitulated in vitro by showing that TL1A stimulation decreased transepithelial electrical resistance (TEER, a measure of paracellular permeability) and increased paracellular permeability of cultured epithelial Caco-2 cells to a small uncharged solute, along with increases in $p$-myosin light-chain kinase (MLC), myeloid differentiation primary response protein (MyD88), and TNF receptor-associated factor (TRAF)6 expression. The authors conclude that TL1A impairs intestinal epithelial barrier function in mice with DSS colitis.

Although the present study does not elaborate on the mechanism of TL1A-mediated disruption of epithelial barrier function, certain hypotheses are worth testing. TL1A/ DR3 may act as an intermediate regulator that increases the 
mucosal concentration of cytokines that impair barrier function. Such proteins include IL-13 and TNF- $\alpha$, which are both upregulated through TL1A/DR3 signaling. In particular, the TL1A-Tg strain that was used by the authors is associated with enrichment of Th-2 lymphocytes of the mucosal compartment with elevations of IL-13 [4], the latter known for its deleterious effect on epithelial integrity, including a positive effect on the expression of the pore-forming tight junction protein claudin-2 [5] that was also observed in the present study. Since TNF- $\alpha$ also increases intestinal paracellular permeability, its association with TL1A may be of importance. In fact, TL1A may act upstream to TNF by inducing its expression [6], a finding that was confirmed in the recent work. Moreover, the addition of TL1A boosted responses to TNF- $\alpha$ in cultured Caco- 2 cells, raising the possibility for a combined negative regulatory effect of TL1A and TNF- $\alpha$ on epithelial barrier integrity. The stimulatory effect of TL1A on Caco-2 cells indicates expression of its receptor by the cultured epithelial cell line. This was also shown in another recent study [7] implying that DR3 should no longer be considered an exclusive lymphocytic marker. Rather, a broader involvement of TL1/DR3 signaling may occur during epithelial crosstalk with either stromal or myeloid cells.

In the present work, constitutive overexpression of TL1A led to severer colitis after DSS administration, pointing to a pro-inflammatory function of TL1A during acute intestinal inflammation. This inflammation-aggravating effect of TL1A was also shown in mice with IL-12+IL-18-induced enterocolitis, which again was associated with epithelial damage [8]. These results, however, need to be interpreted with caution since opposite effects have also been recently demonstrated. Indeed, in a recent study, TL1A-KO and DR3-KO mice suffered from severer DSS colitis, indicating a protective function for these proteins in an acute colitis model [9]. These discrepancies may be explained by differences in the experimental design between the studies. In particular, the model used in the present study involved the sequential application of four cycles of DSS administration that eventually leads to chronic colitis. Similar to the present results, in a prior study, a neutralizing monoclonal antibody against murine TL1A ameliorated chronic DSS colitis [10]. Nonetheless, these models are quite different from the onecycle DSS administration protocol that results in acute, selflimited colitis (and also used in the study by Buttó et al. [9]). It was proposed that TL1A/DR3 exerts dichotomous effects depending on the clinical or experimental situation, being protective against acute mucosal injury but pro-inflammatory during chronic inflammatory conditions, a concept that has been recently validated for several other innate immunity factors.

In conclusion, TL1A and DR3 were originally shown to act as an amplification system for adaptive effector immunity, irrespective of the particular polarization pattern, since they boost Th1, Th2 as well as Th17 responses. Subsequently, they were shown to be equally important for the function of regulatory $\mathrm{T}$ cell responses. Finally, it was demonstrated that TL1A/DR3 are essential regulators of innate immunity, mainly by affecting the function of innate lymphoid cells. In the study by Yang et al., the authors introduce a new potential function of TL1A/DR3 on epithelial cells and the increased paracellular intestinal permeability that is associated with immunological responses in IBD. This finding not only provides a framework to further understand the involvement of TL1A/DR3 signaling in intestinal inflammation, but also adds complexity as their various functions may be interrelated and self-perpetuating (Fig. 1). Further elucidation of such mechanisms will not only help clarify the pathogenic functions of TL1A/DR3 signaling but, most
Fig. 1 The multiple effects of increased TL1A signaling in the amplification of chronic intestinal inflammation

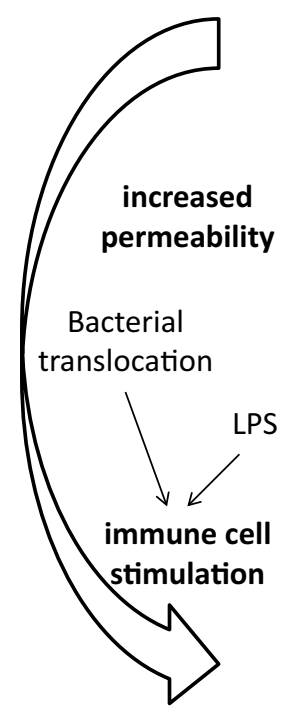

\section{breakdown of epithelial barrier}
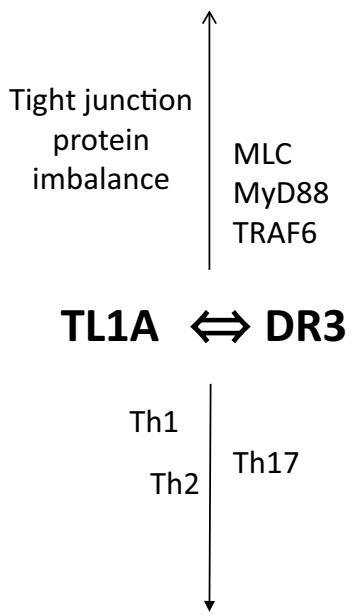

immunological activation

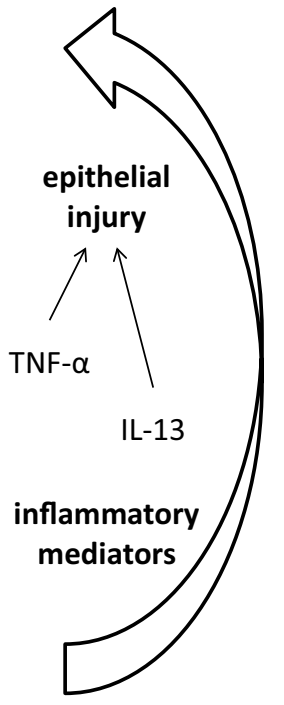


importantly, the therapeutic potential of their manipulation in patients with IBD and other chronic inflammatory conditions.

\section{References}

1. Salim SY, Soderholm JD. Importance of disrupted intestinal barrier in inflammatory bowel diseases. Inflamm Bowel Dis. 2011;17:362-381.

2. Valatas V, Kolios G, Bamias G. TL1A (TNFSF15) and DR3 (TNFRSF25): a co-stimulatory system of cytokines with diverse functions in gut mucosal immunity. Front Immunol. 2019;10:583.

3. Yang M, Jia W, Wang D, et al. Effects and mechanism of constitutive TL1A expression on intestinal mucosal barrier in DSSinduced colitis. Dig Dis Sci. (Epub ahead of print). https://doi. org/10.1007/s10620-019-05580-z.

4. Shih DQ, Barrett R, Zhang X, et al. Constitutive TL1A (TNFSF15) expression on lymphoid or myeloid cells leads to mild intestinal inflammation and fibrosis. PLOS ONE. 2011;6:e16090.

5. Heller F, Florian P, Bojarski C, et al. Interleukin-13 is the key effector Th2 cytokine in ulcerative colitis that affects epithelial tight junctions, apoptosis, and cell restitution. Gastroenterology. 2005;129:550-564.
6. Jin S, Chin J, Seeber S, et al. TL1A/TNFSF15 directly induces proinflammatory cytokines, including TNFalpha, from CD3 + CD161 + T cells to exacerbate gut inflammation. Mucosal Immunol. 2013;6:886-899.

7. Bamias G, Filidou E, Goukos D, et al. Crohn's disease-associated mucosal factors regulate the expression of TNF-like cytokine 1A and its receptors in primary subepithelial intestinal myofibroblasts and intestinal epithelial cells. Transl Res. 2017;180:118-130.

8. Tougaard P, Martinsen LO, Zachariassen LF, et al. TL1A aggravates cytokine-induced acute gut inflammation and potentiates infiltration of intraepithelial natural killer cells in mice. Inflamm Bowel Dis. 2019;25:510-523.

9. Buttó LF, Jia LG, Arseneau KO, et al. Death-domain-receptor 3 deletion normalizes inflammatory gene expression and prevents ileitis in experimental Crohn's disease. Inflamm Bowel Dis. 2019;25:14-26.

10. Takedatsu H, Michelsen KS, Wei B, et al. TL1A (TNFSF15) regulates the development of chronic colitis by modulating both T-helper 1 and T-helper 17 activation. Gastroenterology. 2008;135:552-567.

Publisher's Note Springer Nature remains neutral with regard to jurisdictional claims in published maps and institutional affiliations. 